\title{
MAJOR ARTERIAL INJURY IN CLOSED FRACTURE OF THE NECK OF THE HUMERUS
}

\author{
Report of a Case
}

\section{E. H. J. Smyth, Ryde, ISLe of Wight, England}

A woman aged eighty-six fell in her home sustaining a closed fracture of the neck of the left humerus. Soon after the accident she was seen by her family doctor, who found the whole forearm to be cold and cyanotic with an absent radial pulse. She was sent to hospital, twenty miles away, where on arrival she had signs of complete loss of arterial circulation in forearm, wrist and hand. Radiographs showed a fracture of the neck and great tuberosity of the left humerus, with moderate displacement (Fig. 1).

At operation five hours after the injury an incision was made along the lower part of the delto-pectoral groove, preserving the cephalic vein, and prolonged downwards. The third part of the axillary and upper part of the brachial artery were exposed. The main trunk of

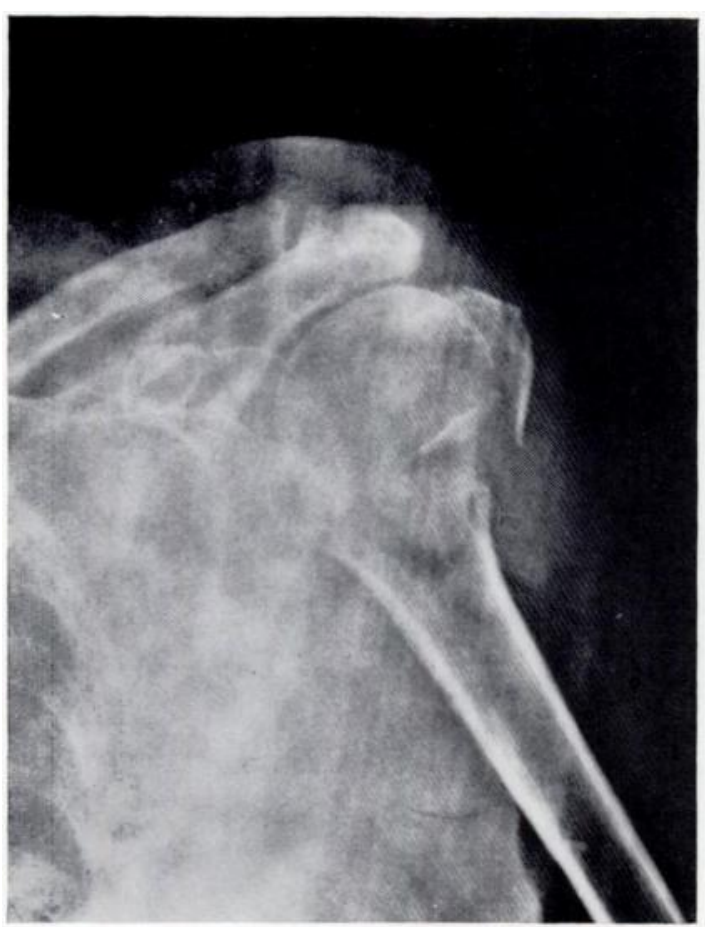

FIG. 1

A radiograph of the fracture. the axillary artery was acutely kinked and pulled laterally at the point of origin of the anterior circumflex artery, which was caught between the fragments of the humerus. Distal to this point, a segment of the axillary trunk 2.5 centimetres in length was seen to be damaged, and was occluded by thrombus; below this point the brachial artery was greatly narrowed by spasm. The anterior circumflex artery was ligated and divided, with immediate release of the kink in the axillary trunk. The axillary artery was opened distal to the thrombosed segment and the clot sucked out, with restoration of flow. The narrowed trunk of the brachial artery was freed from all surrounding soft structures and its lumen was perfused with papaverine solution. Flow was restored well below the injured area, but despite prolonged attempts, including injection of saline into the lumen, the brachial artery spasm remained unrelieved. The artery and wound were closed. Figures 2 and 3 show the findings at operation.

Progress-A line of demarcation appeared just below the elbow, and the forearm, wrist and hand became gangrenous. The patient's general condition remained poor. Twelve days after injury mid-brachial amputation was carried out, after which her condition improved and she made an otherwise good recovery.

\section{DISCUSSION}

Numerous instances of vascular injuries complicating fractures and surgical operations have appeared in the recent literature. This further case seemed worthy of recording because 


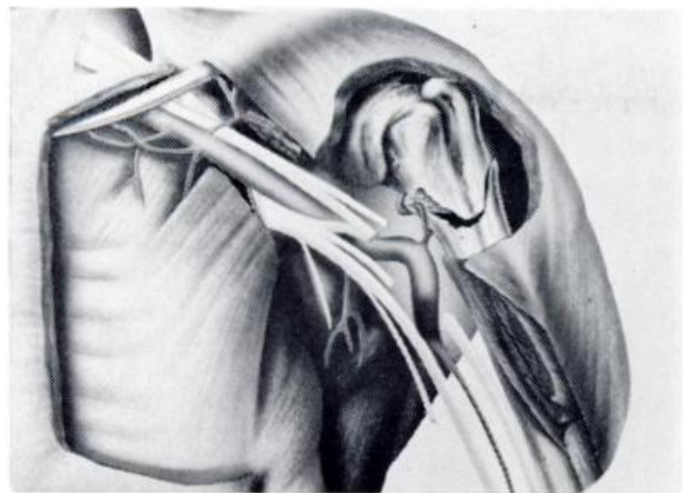

FIG. 2

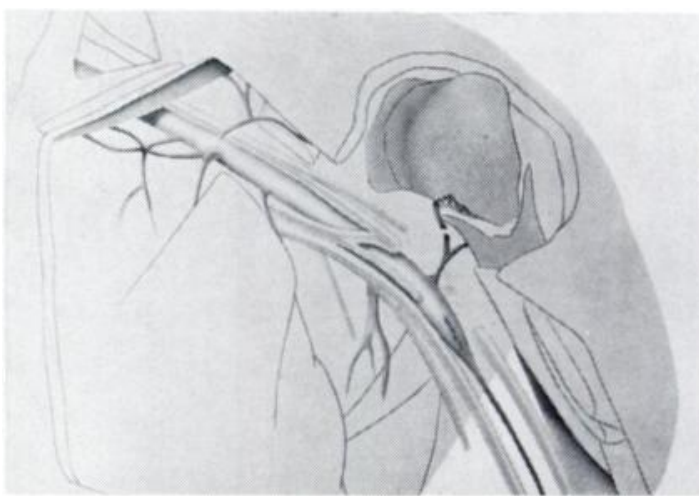

FIG. 3

Operative findings. Figure 2-The initial findings at operation: the anterior circumflex artery had been trapped and pulled forward by the fracture: there was a thrombosed segment of the axillary artery and, distally, spasm of the brachial arterial trunk. Figure 3-After release of the axillary artery, ligation of the anterior circumflex artery, arteriotomy and the removal of thrombus the spasm persisted in the brachial artery.

of the rarity of severe injury to the axillary artery in closed fractures of the neck of the humerus. In a series of 220 acute arterial injuries, 10 per cent in association with fractures, Morris, Beall, Roof and de Bakey (1960) cited no case of vascular injury with a humeral neck fracture, nor any injury of the axillary artery. McQuillan and Nolan (1968) described one case of thrombosis of the axillary artery complicating fracture-dislocation in a woman of seventy-three, in which the whole area around the artery was found at operation to be a mass of fibrous tissue because of previous mastectomy and radiotherapy. Treatment by arteriotomy and thrombectomy was unsuccessful in relieving the ischaemia.

In this case vascular damage was inflicted at the moment of fracture and its effects were apparent to the general practitioner shortly afterwards; on arrival at hospital the signs were those of total ischaemia. Two distinct factors were responsible for occluding the artery: thrombosis from contusion, and spasm from kinking and distortion from the pull of the displaced anterior circumflex artery. Some of these factors were countered by anterior circumflex artery ligation combined with arteriotomy and removal of clot, but distal spasm remained unrelieved despite restoration of a pulsating flow below the contused segment and repeated instillation of papaverine and saline into the lumen.

All authorities agree that exploration of the damaged vessel is indicated as soon as a diagnosis of ischaemia has been made; and that the deep fascia must be split and the arteriai injury dealt with according to circumstances by suture, arteriotomy, endarterectomy or excision of the damaged segment and graft replacement. In our case endarterectomy was not considered necessary in view of the restored distal flow, and it is doubtful if it would in any case have been feasible because, according to Stiles (1965), this procedure is only possible when there exists a suitable plane of separation between intima and media, as found in arteriosclerosis or with longstanding thrombi. Resection-arteriectomy would have been the treatment of choice but was rejected owing to the patient's age and poor general condition.

Arterial spasm has recently been defined by Nolan (1968) as "a response to violent distortion," a state of affairs vividly illustrated in this case, and also significant in view of the incidence of spasm in high velocity missile wounds. Although McQuillan and Nolan (1968) only met with a single instance of definite arterial spasm in their review of thirty-seven cases of traumatic ischaemia, this figure in all probability does not reflect the true incidence. The condition remains so often unrecognised only because it is not more frequently explored. Unfortunately there is still no uniformly successful means of relieving it, and authorities differ as to its management. Seddon (1964) advised, in order of priority, incision of the deep fascia, instillation of $2 !$ per cent papaverine, and excision of the injured segment initiating the spasm.

VOL. 51 B, NO. 3, AUGUST 1969 
He added: "Let us hope that a completely futile sympathetic block will not have been done." Bonney (1963) and others favoured instillation of heparin (1,000 units) into the lumen below the obstruction, but as Eastcott (1965) emphasised, anticoagulants are of limited value in the arterial circulation, because arterial thrombi consist of platelets and lymphocytes rather than fibrin, and in addition the rapid stream is likely to carry them away before they have time to act. In support of this contention Eastcott cited the interesting fact that coronary thrombosis can occur in haemophiliacs. The method of Mustard and Bull (1962), in which the distal arterial tree is opened up by injecting it with saline, segment by segment between clamps, seems always worthy of trial because of its simplicity.

\section{SUMMARY}

A case of injury of the axillary artery complicating a closed fracture of the neck of the humerus in a woman of eighty-six is reported. Spasm was not relieved despite repeated instillation of papaverine. Gangrene developed and amputation above the elbow was required.

I am greatly indebted to Mr R. C. Cole, Chief Instructor, Department of Naval Illustration, H.M.S. Vernon, for the two illustrations in Figures 2 and 3.

\section{REFERENCES}

Bonney, G. (1963): Thrombosis of the Femoral Artery Complicating Fracture of the Femur. Journal of Bone and Joint Surgery, 45-B, 344.

EASTCOTT, H. H. G. (1965): The Management of Arterial Injuries. Journal of Bone and Joint Surgery, 47-B, 394. MCQuillan, W. M., and Nolan, B. (1968): Ischaemia Complicating Injury. Journal of Bone and Joint Surgery, 50-B, 482.

Morris, G. C., Beall, A. C., Roof, W. R., and De BaKey, M. E. (1960): Surgical Experience with 220 Acute Arterial Injuries in Civilian Practice. American Journal of Surgery, 99, 775.

Mustard, W. T., and BulL, C. (1962): A Reliable Method for Relief of Traumatic Vascular Spasm. Annals of Surgery, 155, 339.

Nolan, B. (1968): Vascular Injuries. Journal of the Royal College of Surgeons of Edinburgh, 13, 72.

SEDdon, Sir H. (1964): Volkmann's Ischaemia. British Medical Journal, i, 1587.

Stiles, P. J. (1965): Closed Injuries of the Iliac Arteries. Journal of Bone and Joint Surgery, 47-B, 507. 